\title{
Creencias de docentes de preescolar sobre ciencia y tecnología: desafíos para la apropiación social del conocimiento en la infancia
}

Preschool Teachers' Beliefs about Science and

Technology: Challenges for the Social Appropriation of

Knowledge in Childhood

Crenças de professores de pré-escola sobre ciência e tecnologia: desafios para a apropriação social do conhecimento na infância

\author{
Emily Quevedo-Pinzón* (D) orcid.org/0000-0001-6678-3413 \\ Manuel Franco-Avellaneda** iD orcid.org/0000-0002-0895-8219
}

Para citar este artículo

Quevedo-Pinzón, E. y Franco-Avellaneda, M. (2022). Creencias de docentes de preescolar sobre ciencia y tecnología: desafíos para la apropiación social del conocimiento en la infancia. Revista Colombiana de Educación, 1(84), 1-22. https://doi.org/10.17227/rce.num84-11365

Fecha de recepción: 28/02/2020

* Docente. Correo: emilyquevedoconsultora@gmail.com

** Investigador. Correo: mfrancoavellaneda@gmail.com 


\section{Resumen}

Palabras clave: infancia; creencias; museos; apropiación social de ciencia y tecnología; educación preescolar y primaria; formación docente

Keywords: childhoods; beliefs; social appropriation of science and technology; kindergarten and primary education; training teacher

Palavras-chave: infância; crenças; museus; apropriação social da ciência e da tecnologia; educaçã̃o préescolar e fundamental; formação de professores
En 2019, el museo interactivo Maloka implementó la Ruta de formación de profesores en ciencia y tecnología, en Bogotá (Colombia), una iniciativa para orientar el diseño e implementación de proyectos de innovación pedagógica en instituciones educativas públicas de la ciudad. En este sentido, la Ruta tuvo por objeto desarrollar procesos de formación con docentes para promover la construcción colaborativa de propuestas educativas que usen el Museo para el desarrollo del pensamiento científico y tecnológico de niñas y niños. En este proyecto se realizó una investigación cualitativa con las docentes de preescolar y primero de primaria, que develó algunas de sus creencias sobre las ciencias y las tecnologías, y su relación con las niñas y niños entre 4 y 7 años en la escuela. Las reflexiones evidencian ciertos desafíos a los que se enfrenta la apropiación social de la ciencia y la tecnología cuando se piensa y actúa en clave de infancias.

\section{Abstract}

In 2019, Maloka, an interactive science museum in Bogotá Colombia, implemented the training teacher Route in Science and Technology. This initiative was aimed to guide the design and implementation of pedagogical innovation projects in public schools in Bogotá. This Route developed a training process with teachers to promote the collaborative building of educational proposals for using the Museum to develop experiences that promote scientific and technological thought at school level. In this framework we developed qualitative research focused on kindergarten and primary school teachers. Working with this audience allowed us to understand the beliefs of teachers about the development of scientific knowledge in children between 4 and 7 years old. The comprehension about these beliefs highlight some of the challenges facing the social appropriation of science and technology when thinking and acting from the perspective of childhood at school level.

\section{Resumo}

No ano de 2019, o museu interativo Maloka, em Bogotá, Colômbia, implementou a Rota de Formação de Professores m Ciência e Tecnologia, uma iniciativa que procurava orientar o desenho e a implementação de projetos de inovação pedagógica em escolas da rede pública da cidade. Nesse sentido, o objetivo da Rota foi desenvolver processos de formação com professores para promover a construção colaborativa de propostas educacionais que utilizam o Museu para o desenvolvimento do pensamento científico e tecnológico de meninas e meninos. Neste projeto, foi realizada uma pesquisa qualitativa com professores da pré-escola e do primeiro ano do ensino fundamental, que revelaram algumas das suas crenças sobre ciência e tecnologia, adicionalmente de sua relação com as crianças entre 4 e 7 anos de idade na escola. As reflexões revelam alguns dos desafios enfrentados pela apropriação social da ciência e da tecnologia ao pensar e agir na chave da infância. 


\section{Introducción}

En este artículo recogemos las indagaciones realizadas en el marco de un proyecto de formación de 87 docentes de 17 instituciones educativas de Bogotá, realizado en 2019 en Maloka, museo interactivo de ciencia y tecnología ubicado en esa ciudad. La Ruta de formación en ciencia y tecnología (Ruta), como denominamos el proyecto, se desarrolló en convenio entre la Secretaría de Educación Distrital (SED) y Maloka.

En este texto revisamos la experiencia de las profesoras de preescolar y básica primaria, quienes fueron priorizadas en el proyecto con el argumento de que existe una gran necesidad de formación de docentes en ciencia y tecnología, especialmente en grados iniciales, por el desafío que enfrentan al enseñar todas las áreas dejan las ciencias y tecnologías relegadas para años superiores de escolaridad. Esto bajo premisas tales como "no es necesario enseñar tan temprano estos contenidos" o "porque las profesoras no se sienten autorizadas para enseñar ciencias, pues se trata de un conocimiento especializado".

Bajo ese entendido, nuestra investigación indaga y devela la relación de algunas de las creencias que las docentes de preescolar y primero tienen sobre la ciencia y la tecnología, y los posibles desafíos que estas pueden ocasionar en la apropiación social de la ciencia y la tecnología en las niñas y niños que asisten a estos grados escolares.

Antes de comenzar, es fundamental entender la naturaleza del lugar desde el que se desarrolló la formación, en especial porque estos centros de ciencia, donde se incluyen los museos interactivos, son entendidos en la política pública colombiana como actores del sistema nacional de ciencia, tecnología e innovación, pues son responsables de promover la apropiación social de la ciencia, la tecnología y la innovación (ASCTI) ${ }^{1}$. Estos espacios desde su nacimiento desarrollan acciones articuladas con la educación básica y poco a poco se amplían a la educación inicial, media y universitaria, a la vez que se involucran con diferentes actores del sistema educativo.

Dichos museos tienen su origen a finales de la década de 1960, y podrían caracterizarse por lo menos con tres cambios que tienen relación directa con la educación, en concordancia con el trabajo de Schiele (2008) y Franco-Avellaneda (2013a; 2013b), así: la comunicación, la interactividad y la evaluación.

Asociados con la comunicación y la diferencia de los museos de ciencias naturales del siglo XIX, que proponían una comunicación entre expertos, estos escenarios proponen un cambio para una comunicación centrada en los públicos, justificado en la aparente imposibilidad de los científicos de comunicarse con la sociedad y la incapacidad de compartir el inmenso poder que tienen los conocimientos científicos (Beetlestone et al., 1998).

La interactividad se articula con el creciente interés de la época por transformar el sistema educativo, el cual se consideraba, por un lado, aislado de las necesidades e intereses de la sociedad y, por el otro, necesitado de un aprendizaje basado en la experiencia y no en los contenidos (Yager y Penick, 1983). Esto está articulado con la llamada crisis de la educación

\footnotetext{
${ }^{1}$ La política pública ha definido la apropiación social de la ciencia, la tecnología e innovación como "un proceso intencionado de comprensión e intervención en las relaciones entre ciencia, tecnología y sociedad, que tiene como objetivo ampliar las dinámicas de generación, circulación y uso del conocimiento científico-tecnológico, y propiciar las sinergias entre sectores académicos, productivos, estatales, incluyendo activamente a las comunidades y grupos de interés de la sociedad civil" (DNP-Colciencias, 2015, p. 20).
}

Primer cuatrimestre de 2022 (enero-abril) || e-ISSN: 2323-0134 || 3 
(Coombs, 1971) y el posicionamiento de las otras educaciones (no formal e informal). Así, estos espacios se erigen como alternativos a la escuela, que es vista como extremadamente libresca, con currículos inflexibles y con poca o ninguna infraestructura para la enseñanza de ciencias y tecnologías (Franco-Avellaneda y Von-Linsingen, 2011; Massarani y Moreira, 2009).

En cuanto a la evaluación, esta se entiende como un proceso mediante el cual se obtiene y analiza información de los públicos del museo, con el objetivo de cualificar sus propósitos. Así, existe un cambio de una evaluación con el objetivo de verificar hacia una evaluación formativa (Screven, 1990). Esto tiene relación con la configuración de nuevo papel para el museo, pues este se convertiría en un lugar de mediación de conocimientos, usando la interactividad para optimizar la comunicación y consecuentemente conseguir que los visitantes participen activa y voluntariamente de sus exposiciones, situación que pone de manifiesto su dimensión educativa.

El posicionamiento de los museos interactivos como alternativos a la escuela $-y$ en ocasiones como competidores de esta, en la medida que también pretenden una democratización del conocimiento, en este caso tecnocientífico- encerraba una contradicción. Estos espacios tienen vida porque existe la escuela; de hecho, gran parte de los recursos proviene de actores relacionados con el sistema educativo, pues el público escolar es el que más frecuenta estos escenarios. Como lo muestra el trabajo de Pérez-Bustos (2009), los museos interactivos, al tomar distancia del escenario escolarizado, terminan por incorporarse al sistema educativo.

Paralelo al desarrollo de los museos, se encuentra, entonces, la necesidad de una formación específica en ciencia y tecnología en las maestras, particularmente en las de preescolar y primero de primaria, que viene siendo abordada tímida y desarticuladamente por algunos actores tanto del campo de la ciencia y tecnología, como de la educación inicial y primaria. En el caso particular del primer campo existen algunos desarrollos en la enseñanza de las ciencias y la tecnología que plantean reflexiones acerca de la formación de estas docentes, vislumbrando la necesidad de incorporar en la cualificación docente esta área de conocimiento, para garantizar la mediación de las maestras en la apropiación social de la ciencia y la tecnología (Arana et al., 2006).

Por su parte, el campo de la educación inicial ha avanzado en reconocer que el pensamiento científico y tecnológico forma parte del desarrollo integral de las niñas y niños (Ministerio de Educación Nacional-MEN, 2014) y, en consecuencia, es un conjunto de saberes que debe ser manejado por las docentes que se encargan de este ciclo vital. Allí, los principales hallazgos se centran en encontrar un equilibrio en las instancias formativas a docentes para que la apropiación de la ciencia y la tecnología no se convierta en la enseñanza de una disciplina, sino más bien en la posibilidad de potenciar habilidades de pensamiento científico y tecnológico desde las particularidades educativas y didácticas de las niñas y niños de 4 a 6 años (Kaufmann y Serulnicoff, 2000).

Para el desarrollo del artículo partimos de describir la ruta de formación y algunas particularidades de las docentes que participaron en esta. Después, abordamos las comprensiones conceptuales que sirven como punto de partida del trabajo, presentando el conocimiento profesoral, la apropiación de la ciencia y tecnología en la infancia y algunos desarrollos del Estado colombiano que la viabilizan en la primera infancia como soporte teórico de la investigación. 
En seguida, describimos las consideraciones metodológicas con las que se desarrolló el estudio y las creencias identificadas en los docentes y sus posibles desafíos en la apropiación social de la ciencia y la tecnología en la primera infancia. Por último, compartimos unas consideraciones finales, las cuales recogen los principales hallazgos y abren nuevas preguntas de investigación.

\section{La ruta de formación en ciencia y tecnología a docentes: ¿cómo se configuró?}

Se constituyó como un proceso de formación cuyo eje fue la conformación de grupos de docentes por instituciones educativas distritales (IED), para la creación e implementación de proyectos de innovación pedagógica (PIP). Esta última fue entendida como un acto deliberado y planificado de solución de problemas de las instituciones, a través de un proceso de construcción de sentido sobre la práctica pedagógica, que la interroga y crea nuevas posibilidades de saber-hacer (Barraza, 2005).

Desde allí, la Ruta se propuso: a) fortalecer conocimientos sobre la relación museo/escuela y su potencial como generador de innovación pedagógica; b) promover la apropiación de espacios de la ciudad como escenarios pedagógicos, y c) proponer diferentes usos de la ciencia y la tecnología para posibilitar proyectos de innovación pedagógica (Maloka, 2019a).

Para alcanzar estos propósitos, grupos de cinco docentes aproximadamente por cada IED desarrollaron un PIP que atendiera algunas necesidades de la vida cotidiana de la escuela. La ideación, diseño e implementación de estos proyectos se desarrolló entre abril y noviembre de 2019. Esta labor se realizó colaborativamente entre los docentes y el equipo pedagógico de Maloka -compuesto por once profesionales con experiencia en educación y ciencia y tecnología-. La formación y el acompañamiento ejecutado en la Ruta con las y los docentes se implementó con la siguiente metodología:

- Formaciones presenciales. Espacios de formación en el museo donde, a través de talleres se intercambiaron y enriquecieron saberes y experiencias en relación con ciencias, tecnologías e innovación pedagógica, el diseño e implementación, seguimiento y evaluación de un PIP. Estas formaciones presenciales se realizaban cada cuatro o cinco semanas. En total se desarrollaron nueve formaciones de cinco horas cada una.

- Acompañamiento situado. Fueron espacios entre las semanas de formación presencial donde el equipo pedagógico de Maloka asistía a las IED y desarrollaba procesos de construcción colectiva, orientación e interacción con todos los docentes de cada institución para la exploración de diversos usos de conocimiento científico y tecnológico en los PIP.

- Actividades de autoformación. La Ruta tuvo énfasis en el reconocimiento de la capacidad de agencia de todos los docentes en su formación; por ello, la autoformación fue uno de los ejes de la Ruta. Allí, a través de guías pedagógicas orientadoras, se promovía el trabajo colaborativo para que como colectivo los docentes por IED avanzaran en la ideación, diseño, implementación y seguimiento de su PIP. 
- Intercambio entre pares. Se generaron espacios con investigadores en educación, en donde los docentes intercambiaban con especialistas sus desafíos y posibilidades de desarrollo del proyecto.

Esta propuesta de formación contó con la participación de 17 instituciones educativas de Bogotá (Maloka, 2019b), distribuidas geográficamente en diferentes localidades: tres en Bosa, dos en Chapinero, una en Ciudad Bolívar, dos en Engativá, una en Fontibón, una en Kennedy, dos en Rafael Uribe, dos en San Cristóbal y tres en Usme. Este conjunto de instituciones educativas conformó un grupo de 87 docentes participantes.

En promedio, los y las docentes que participaron en la Ruta llevaban 7,4 años vinculados a la IED en la que enseñaban, una vinculación con el sector público de 10,8 años y una experiencia en docencia de 18,3 años (Maloka 2019c). Es decir, en general, los participantes eran profesionales experimentados en el campo educativo y en el sector público. El 97,7\% contaba con pregrado y un $72 \%$ con un título de posgrado (maestría 45,3\% y 26,7\% especialización). Además, es importante señalar que 37,64 \% de las docentes están formadas en licenciaturas de educación básica (18,82\%), preescolar y/o infantil $(18,82 \%)$, que corresponde a un total de 43 personas.

En el caso de las docentes de preescolar y primero, hablamos de un total de 14 personas cuya experiencia en la Ruta se convierte en el eje del presente artículo.

\section{El punto de partida conceptual}

Para el desarrollo teórico se retoman tres lugares de enunciación: a) el conocimiento profesoral que da contexto a las creencias de los docentes; b) la apropiación de la ciencia y la tecnología en la infancia, y c) algunas comprensiones del Estado colombiano sobre la primera infancia y su relación con la ASCTI.

En primer lugar, desde los años 1980 se ha desarrollado una corriente de estudios denominados conocimiento profesoral, desde estos se afirma que los docentes en su quehacer cotidiano de enseñanza no se limitan únicamente al uso de saberes disciplinares sobre el área en la que trabajan, sino también a un cuerpo de conocimientos en los que se conjugan la experiencia, la didáctica, la metodología y el conocimiento de sus estudiantes del contexto, con la formación disciplinar de su especialidad (Valbuena, 2007).

En ese sentido, Shulman (1987) identificó que el conocimiento del profesor puede tener siete tipos: disciplinar, pedagógico, del currículum, de los alumnos y del aprendizaje, del contexto, didáctico del contenido y de filosofía educativa. En paralelo a los trabajos de Shulman, se encuentran los de Grossman (1990), quien hace su propia categorización al señalar que el pedagógico general, el del contexto y el didáctico del contenido son los componentes esenciales del conocimiento del profesor.

Además de esta composición de los saberes de conocimiento profesoral, Magnusson et al. (1999) incorporaron a la caracterización el concepto de concepciones, que definieron como aquellas ideas que tienen los docentes acerca del conocimiento profesional; es decir, los saberes con los que operan las docentes no solo están compuestos por los saberes disciplinares, sino también por las experiencias y los intereses de cada uno. Estos autores afirman, además, que estas concepciones tienden a tener mayor arraigo en las docentes, y 
por tanto son difíciles de transformar; paralelamente, no se recurre a las concepciones de manera ocasional, sino que estas influyen de manera directa en la toma decisiones de la enseñanza y por esto deben considerarse al mismo nivel que los conocimientos de sus áreas de formación.

Teniendo en cuenta estas comprensiones del conocimiento profesoral, para nuestro interés sobre la Ruta será importante detenernos en las creencias de los profesores, entendidas estas como esos saberes del docente que integran diferentes variables: disciplinares, didácticas, experienciales, intereses e imaginarios del docente, así como sus concepciones sobre el saber y la enseñanza. Lo anterior, dado que nuestra investigación con las docentes de preescolar y participantes de la Ruta devela que las creencias de las docentes sobre las ciencias y tecnologías son determinantes en los procesos de AScTI con las niñas y niños.

Para hacerlo, es importante señalar que en Colombia la corriente de estudios del conocimiento profesoral ha sido abordada por varios trabajos (Arana et al., 2006; Ruiz et al., 2009). En estos dos estudios, los hallazgos más relevantes están relacionados con la identificación del poder de las concepciones y creencias que sobre ciencias y tecnologías tienen los docentes para la enseñanza de estas, tanto en los que cuentan con una formación base en educación infantil, así como en los docentes con formación disciplinar especializada.

En el caso de Arana et al. (2006), su investigación identificó que los docentes de preescolar investigados manifiestan una percepción de las ciencias alejada de su función social, igualmente perciben las tecnologías ligadas a dispositivos tecnológicos; en consecuencia, existe la creencia de que abordar ciencia y tecnología con las niñas y niños requiere condiciones económicas ligadas a los objetos y materiales, puesto que se requiere invertir en estos para desarrollar estos conocimientos en los procesos educativos.

En cuanto a la investigación de Ruiz et al. (2009), se encontró que los docentes tienen la tendencia a creer que las ciencias son rígidas y se limitan al desarrollo de un método; además, la identifican como una disciplina sin rol social, es decir, no ven la relación de las ciencias y tecnologías con procesos históricos, políticos ni sociales. Al ser tan formal, consideran que es indispensable que se realicen procesos de formación que preserven esta condición fidedigna del conocimiento científico. Respecto a la tecnología, coinciden con la investigación de Arana et al. (2006), pues la identifican como un asunto de aplicación de conocimientos en la construcción de artefactos.

Estos hallazgos concuerdan con las investigaciones desarrolladas por Fernández et al. (2002), Gallego (2007) y Franco-Avellaneda y Pérez-Bustos (2009), quienes encontraron visiones deformadas de ciencia presentes en los textos escolares, cómics y textos de divulgación científica, que consideramos pueden relacionarse con concepciones que tienen los docentes, pues los libros didácticos terminan influenciando su comprensión de ciencias y tecnologías.

En efecto, Fernández et al. (2002) afirman que la incidencia de las concepciones de los docentes es trascendental en la didáctica de la enseñanza del conocimiento científico. Particularmente, dentro de estas investigaciones señalan siete concepciones deformadas que son trasmitidas en la enseñanza por los docentes: empiro-inductista y atéorica, rígida de la actividad científica, aproblemática y ahistórica de la ciencia, exclusivamente analítica, meramente acumulativa del desarrollo científico, individualista y elitista de la ciencia, y la visión descontextualizada socialmente neutra de la actividad científica. 


\section{Apropiación de la ciencia y la tecnología en la infancia: más proceso que resultado}

La apropiación social de la ciencia y la tecnología es un término que se usa en la política pública colombiana desde la década de 1990 como alternativa a palabras como divulgación, popularización y vulgarización, los cuales se asocian semánticamente con la idea de llevar a los ciudadanos conocimientos en una lógica deficitaria -desde el que sabe para el que no sabe (Franco-Avellaneda, 2016)-. En ese sentido, la apropiación, más allá de la definición institucional presentada en la introducción, promovería una participación activa del ciudadano en la generación, uso y circulación del conocimiento científico y tecnológico.

Así, se superarían los determinismos tecnológico y social para hablar de una ciudadanía sociotécnica como eje para ser y actuar en las sociedades actuales. Esto, reconociendo que las ciencias y tecnologías no son neutrales y, por ende, existen alternativas científico-técnicas para elegir, porque estas opciones constituyen la base material que determina la viabilidad de ciertos modelos socioeconómicos y políticos, así como la imposibilidad de otros. Por tanto, parece necesario incorporar ciencias y tecnologías como un aspecto fundamental de nuestros sistemas de deliberación política (Thomas, 2009).

A partir de esa comprensión, en términos educativos la apropiación pondría el énfasis en el qué y el para qué, más allá del cómo. En ese sentido, sería un complemento clave para un sistema educativo centrado en la relación funcional del proceso/producto que se enfoca en la eficacia de la enseñanza al mirar la acción del docente (proceso) y el impacto que esta tiene en el aprendizaje (producto). Así las cosas, el desafío para un escenario como Maloka debería ser, identificar necesidades e intereses de apropiación de conocimientos científicos y tecnológicos e incorporarlos a las actividades desarrolladas por el museo.

Como complemento de lo anterior, y en coherencia con la noción de apropiación que usamos, el papel educativo que defendemos para el museo está dirigido a permitir la discusión, negociación e intercambio de ciencias y tecnologías en construcción; es decir, lo educativo permitiría la reflexividad y el aumento de conciencia de los actores implicados en una situación problema (Freire, 2005), esto sería posible gracias a que el museo presentaría y abordaría las ciencias y tecnologías en acción (Latour, 1987).

Esto está relacionado con los planteamientos de Furman (2008) sobre las dos caras de las ciencias. Por un lado, identifica que las ciencias implican un producto, un conjunto de conocimientos, aquello que se sabe y forma parte del acervo construido por científicos en los últimos siglos. Esta cara de producto son las ciencias más presentadas en las escuelas. De otro lado, las ciencias son un proceso, un modo de explorar y comprender la realidad, a través del cual se genera ese conocimiento. Luego, en las ciencias como proceso, cumplen un papel fundamental el pensamiento lógico, la creatividad, la identificación de evidencias, el análisis empírico, la construcción de modelos teóricos y la discusión en un colectivo que trabaja para generar nuevo conocimiento. Esta dimensión de las ciencias es la que suele estar ausente en las escuelas. 
Ahora bien, en el caso específico de la educación inicial, diversas investigaciones han mostrado el poco conocimiento de conceptos científicos que tienen las maestras y maestros (Kelly, 2000; Parker y Heywood, 2000). No obstante, existen estudios que critican esos trabajos, los cuales argumentan que están basados en un paradigma de déficit del profesor (Lima y Maués, 2006), pues asumen que el saber del profesor puede ser modificado para lograr un conocimiento apropiado y que después podría ser transferido a niños y niñas. Estas críticas se basan en trabajos donde se evidencia que, aunque algunos maestros tienen un pobre conocimiento del contenido científico, logran enseñar ciencias satisfactoriamente (Appleton y Kindt, 2002; Maués, 2003); en especial porque recurren a estrategias eficaces para promover y desarrollar aprendizajes en niños y niñas, pues generan actividades significativas, estimulan la creatividad, favorecen interacciones con el mundo y confrontan el conocimiento de sus estudiantes.

En ese sentido, entendemos los contenidos escolares en tres dimensiones conceptuales, procedimentales y actitudinales (Delors, 1996; Lima y Maués, 2006). Los contenidos conceptuales están asociados a los conceptos científicos (saber qué enseñar); los procedimentales están relacionados con el saber hacer (saber enseñar), y los contenidos actitudinales hacen referencia al saber ser con los estudiantes. Así las cosas, cuando se argumenta como dificultad el poco conocimiento en ciencias que tienen las maestras de primera infancia, se hace énfasis en la primera dimensión y se desconocen las otras dos. En efecto, la ciencia como proceso se sustenta en observar, clasificar, probar, argumentar, etc. Esto exige que las profesoras tengan conocimientos o saberes que no son necesariamente de la dimensión conceptual, pero sí del conocimiento de las etapas de desarrollo en las que se encuentran las niñas y niños. Luego, son más del campo de las dimensiones procedimentales y actitudinales, porque implican un saber enseñar y saber ser con sus estudiantes. En esos campos, las profesoras de educación inicial tienen mucho por decir.

\section{Algunas nociones del Estado colombiano sobre primera infancia y su relación con la ASCTI}

En los últimos veinte años, Colombia se ha insertado en un proceso de cualificación y fortalecimiento de los procesos de educación inicial y preescolar, iniciativa que ha estado marcada por el cambio de paradigma respecto a la comprensión de los derechos de las niñas y niños desde su gestación y hasta los 6 años, y particularmente acerca de las transformaciones en las concepciones que sobre ellas y ellos se tiene respecto a ser sujeto de derechos. Desde 2010, esta metamorfosis ha sido liderada y consolidada por diferentes entidades nacionales, particularmente por la Comisión Intersectorial para la Atención Integral a la Primera Infancia (CIPI), el Ministerio de Educación Nacional (MEN) y el Instituto Colombiano de Bienestar Familiar (ICBF), quienes en uso de sus funciones y en cumplimento de la Ley de Infancia y Adolescencia (1098 de 2006) han establecido la política de Estado para el desarrollo integral de la primera infancia de cero a siempre (Ley 1804 de 2016).

Estos desarrollos legislativos señalan que todas las acciones que movilice el Estado gobierno, familia y sociedad civil- respecto a esta población deben tener como fundamento la concepción de niño y niña como seres libres, sociales y ciudadanos sujetos de derechos (CIPI, 2013). Asimismo, establece que las niñas y niños que se encuentran en este ciclo vital están 
en una de sus máximas etapas de desarrollo, por tanto, se apuesta por establecer escenarios en los que se promueva su desarrollo integral (CIPI, 2013). Para ello, el MEN (2017) ha propuesto las Bases curriculares para la educación inicial y el preescolar, las cuales señalan que la labor de los docentes debe centrarse en el impulso de vivencias pedagógicas que potencien propósitos de desarrollo y aprendizaje particulares, y correspondientes a los procesos individuales de las niñas y niños Así, propone que se diseñen, en los entornos educativos donde atienden a esta población, propuestas cuyo currículo se base en la experiencia, así como en el uso de las actividades rectoras: juego, literatura, exploración del medio y literatura.

En particular, para este artículo es relevante detenerse en la actividad rectora de la exploración del medio y en el tercer propósito de desarrollo y aprendizaje, puesto que los dos apuestan por el fortalecimiento del pensamiento científico y tecnológico.

Respecto a la primera, la exploración del medio, el MEN (2014) la define así:

una de las actividades más características de las niñas y los niños en la primera infancia. Al observarlos, se puede ver que permanentemente están tocando, probando, experimentando y explorando todo cuanto les rodea; ellas y ellos están en una constante búsqueda de comprender y conocer el mundo [...]. Esta experiencia de actuar y de relacionarse en el tiempo y en el espacio con las personas, objetos, situaciones, sucesos y contextos, propicia un proceso de construcción de sentido de lo que es y pasa en el mundo, y de lo que implica habitar en él. (MEN, 2014)

Esto se acerca a lo definido por Furman (2016), en relación con los atributos del pensamiento científico, quien lo define como "una manera de pararse ante el mundo, que combina componentes cognitivos y socioemocionales, como la apertura y la objetividad, la curiosidad y la capacidad de asombro, la flexibilidad y el escepticismo, y la capacidad de colaborar y crear con otros" (p. 17).

Esta definición, además de corresponder con las comprensiones del MEN de la actividad rectora de exploración del medio, se relaciona con los propósitos de la educación inicial establecidos por esta misma entidad:

1. Los niños y las niñas construyen su identidad en relación con los otros; se sienten queridos y valoran positivamente pertenecer a una familia, cultura y mundo.

2. Los niños y las niñas son comunicadores activos de sus ideas, sentimientos y emociones; expresan, imaginan y representan su realidad.

3. Los niños y las niñas disfrutan aprender y explorar, y se relacionan con el mundo para comprenderlo y construirlo.

El último propósito se centra en estas posibilidades y capacidades que promueve el pensamiento científico. Estos referentes técnicos guardan relación con los Ilamados derechos básicos de aprendizaje (DBA) del MEN, tanto para el grado transición (2016a) como los DBA de ciencias naturales para el grado primero (2016b). Son así recursos institucionales para la apropiación social de la ciencia y la tecnología desde la primera infancia y consecuentemente un desafío para los docentes que se desempeñan en estos niveles. 


\section{Consideraciones metodológicas}

Una vez que la Ruta comenzó su proceso de implementación, seguimiento y documentación, surgió la necesidad de reflexionar sobre algunas creencias y concepciones de las docentes de preescolar y primaria sobre las ciencias y las tecnologías, y las niñas y niños entre 4 y 7 años en la escuela. Lo anterior, dado que develar las visiones de ciencia que circulan en las concepciones de las docentes no solo animaba la pregunta de nuestra investigación, sino que también movilizaba el proceso de formación docente que gestionaba la Ruta como mecanismo para promover la apropiación social de la ciencia y la tecnología en la infancia.

Para dar cuenta de ello, planteamos una investigación cualitativa. Según Bonilla-Castro y Rodríguez (2005), tiene entre sus premisas el hecho de que la fuente de datos es una situación social y estos son recogidos en condiciones específicas. En ella, el proceso es más valorado que el producto, y por tanto su preocupación no es medir sino describir y analizar el fenómeno (en este caso educativo) en la búsqueda de comprender la forma en que se configuran relaciones y significados por los sujetos involucrados.

En ese sentido, en la investigación que aquí presentamos importa indagar y develar la relación de algunas de las creencias que las docentes de preescolar y primero tienen sobre la ciencia y la tecnología y los posibles desafíos que estas pueden ocasionar en la apropiación social de la ciencia y la tecnología en las niñas y niños. Con este objetivo, la investigación cualitativa nos resulta la más adecuada.

Para ello la recolección de información, que corresponde a los datos empíricos principales, la realizamos a través de los siguientes aspectos:

- La revisión de los PPI. Estos eran desarrollados por las profesoras en sus instituciones con niñas y niños de preescolar y primero de primaria; en total revisamos nueve proyectos pedagógicos de la misma cantidad de IED. El objetivo de este análisis fue hallar las decisiones, propuestas, estrategias que las docentes planteaban en los PIP para la apropiación social de la ciencia y tecnología en niñas y niños de preescolar y primero.

- Grupos focales con los profesionales de Maloka que acompañaban el proceso de cada IED, en estos encuentros se conversó alrededor de cuatro ejes:

- La experiencia de las docentes de preescolar y primero en los PIP y en la Ruta.

- La experiencia (aciertos, fortalezas, aprendizajes, aspectos a fortalecer) de los profesionales de Maloka en el acompañamiento que realizaban a los grados preescolar y primero.

- Las percepciones sobre las relaciones entre apropiación de la ciencia y la tecnología, y la primera infancia.

Establecer la conversación de los grupos focales desde estos ejes tenía como propósito, develar las concepciones que sobre las docentes de preescolar y primero tenían los profesionales de Maloka, así como hallar sus propias concepciones alrededor de la apropiación de la ciencia y la tecnología en estos grados. 
- Encuestas y entrevistas particulares a docentes que trabajan de manera directa con niñas y niños de los grados ya mencionados. Las cuestiones sobre las que giraron estos instrumentos fueron estas:

- Principales imaginarios acerca de la enseñanza de la ciencia y la tecnología en el preescolar y en primero, antes de estar en la Ruta de innovación pedagógica con Maloka.

- Transformaciones de imaginarios de las docentes, teniendo en cuenta la formación y acompañamiento ofrecido por la Ruta y la vivencia en el Museo Maloka.

- Desafíos en labor docente para promover el pensamiento científico y tecnológico con las niñas y niños de primera infancia.

Estas entrevistas y encuestas no solo tenían por objeto encontrar las concepciones que sobre la apropiación de la ciencia y la tecnología tenían las docentes, sino que también aportaban la identificación de percepciones que las docentes tenían sobre el proceso de formación de la Ruta y sus impactos en las relaciones que hasta el momento tenían en la enseñanza de las ciencias y la tecnología con niñas y niños de 4 a 7 años.

Además de esas fuentes empíricas, se realizó revisión a los documentos técnicos que tuvo la Ruta en su desarrollo, específicamente el documento orientador, la caracterización de docentes y los informes de avance de del proceso ${ }^{2}$.

Con el acopio de esta información realizamos el análisis categorial, en el que buscamos asegurar la confiabilidad de la investigación cualitativa a partir de la triangulación de los datos obtenidos por las fuentes y la confrontación con el referencial teórico construido. Así, los resultados que aquí presentamos se derivan del diálogo entre los datos recogidos, la revisión teórica y las principales tendencias que surgían en las docentes.

Nuestro punto de partida es que las visiones sobre las ciencias y tecnologías, así como las comprensiones de infancia, afectan los procesos de enseñanza, específicamente los de apropiación social de la ciencia y tecnología, en la medida en que funcionan como una especie de obstáculo epistemológico en los términos propuestos por Bachelard (2000), como una barrera que limita nuevas comprensiones.

A continuación, presentamos los principales hallazgos del análisis, los cuales están organizados en tres niveles: creencias sobre las niñas y los niños, y sus posibilidades con la ciencia y la tecnología; creencias sobre las profesoras de preescolar y primero al enseñar estos conocimientos, y creencias sobre la disposición de las docentes hacia las ciencias y tecnologías.

\footnotetext{
${ }^{2}$ Como la Ruta de formación en ciencia y tecnología forma parte del convenio 2613-2017, entre la Secretaría de Educación del Distrito y la Corporación Maloka de Ciencia, Tecnología e Información, se elaboraron tres informes de avance de la Ruta a la fecha de publicación de este artículo.
} 


\section{Creencias sobre niñas y niños y sus posibilidades con la ciencia y la tecnología}

A lo largo del proceso de análisis de la información sobre la experiencia de niñas y niños de preescolar y primero en la Ruta, fue recurrente ver cómo las apreciaciones, miedos, conclusiones o necesidades de las docentes o profesionales se relacionaban con la mirada o concepción de las infancias. Al respecto, un grupo considerable de docentes manifestó tener imaginarios sobre las posibilidades de las niñas y niños con la ciencia y la tecnología desde la carencia y la imposibilidad. Estos imaginarios están relacionados con la creencia de que las y los pequeños no están preparados para acceder al conocimiento científico, pues son para personas con más edad.

Estas afirmaciones son las que De la Jara (2015) señala como una definición de las infancias desde la minusvalía intelectual. La autora indica que este concepto está referido a la comprensión sobre la cual niñas y niños están en una situación de desventaja que se deriva por su edad. Refiere que las personas perciben la edad como una limitante a la hora de vivir una experiencia -en este caso, con ciencias y tecnologías- porque el pensamiento, el lenguaje (verbal o escrito), el comportamiento y las habilidades motoras de las y los pequeños al encontrarse en desarrollo generan restricciones a la hora de acceder a experiencias tecnocientíficas. En esta noción de las infancias, niñas y niños son una suerte de obra en elaboración, y las ciencias y tecnologías un saber que solo es posible usar cuando la obra está terminada.

Una de las situaciones que permiten dar cuenta de esta reflexión se vivenció en la visita de niñas y niños de las IED a Maloka, quienes como parte del recorrido al museo interactivo ingresaban al laboratorio Pequeños Inventores ${ }^{3}$ y allí realizaban un taller cuyo objetivo era construir una catapulta, esto como excusa para trabajar habilidades de pensamiento científico y tecnológico. Un número importante de maestras que acompañaban el taller señalaron estar sorprendidas con lo que sus estudiantes realizaron, pues nunca los habían visto hacer este tipo de creaciones; asimismo, la sorpresa estaba relacionada con su capacidad para elaborar la catapulta.

Estas bajas expectativas también fueron identificadas por el equipo pedagógico de Maloka que desarrolló la Ruta, quienes reconocieron que una de las principales barreras que encontraban a la hora de trabajar con las docentes de preescolar y primero se relacionaban con el imaginario sobre niñas y niños como sujetos con dificultades para ejecutar actividades en donde se involucraba el conocimiento científico. Afirmaciones y preguntas como las siguientes, antes de la visita a Maloka, resumen la situación:

- iEsto es muy elevado para los niños de primero!

${ }^{3}$ El laboratorio Pequeños Inventores es un espacio ideado para ofrecer experiencias interactivas de ciencia y tecnología para un público entre 3 y 7 años. En el momento en que se desarrolló la Ruta se encontraba en terminación; sin embargo, ya ofrecía al público de primera infancia una oferta especializada. 
- ¿Y allá (en Maloka) qué van a ir a hacer los niños pequeños?

- ¿Y eso cómo se hace con los niños de preescolar?

Esto también sucedió en el equipo de Maloka, en ese sentido uno de sus miembros señaló:

Yo inconscientemente como facilitadora también subestimaba a los niños de primera infancia, para mí fue un reto cuando vinieron a Maloka, tenía todos los temores del mundo: que los mocos, la lonchera... todo al final fue bien. También rompí un montón de miedos alrededor de qué enseñarles, qué decirles. Fue una experiencia gratificante transformar esos imaginarios. (Facilitadora 1)

Este grupo de creencias refleja cómo la concepción de infancia está ligada a la concepción rígida de la actividad científica señalada por Fernández et al. (2002), para quienes que la ciencia es un cuerpo exacto de conocimiento, infalible. Al ser comprendido así por las docentes, se vuelve una condición determinante para mantener alejadas(os) a las niñas y niños de las experiencias de ciencia y tecnología, pues requieren ser más grandes, maduros y con mayor desarrollo para comprender ese saber exacto que es conocimiento científico y tecnológico.

\section{Creencias sobre las profesoras de preescolar y primero al enseñar ciencias y tecnologías}

Otro de los hallazgos en esta vivencia de las profesoras de preescolar y primero que visibilizó la Ruta está relacionado con las creencias que sobre ellas tienen docentes de ciencias de las IED y los profesionales de Maloka.

En una entrevista a docentes de ciencias de secundaria de una de las IED que realizó un PIP con niñas y niños de preescolar, se señaló que una de las mayores dificultades a la hora de diseñar proyectos de ciencias y tecnologías para los grados de preescolar y primero es la falta de conocimiento científico de las docentes de estos grados. En este sentido, uno de los profesores señaló:

El profe de preescolar puede tener problemas de alfabetización científica y sería muy bueno cerrar esa brecha. Se puede pensar en un proyecto de IED, pero se tiene que trabajar en el desarrollo de habilidades científicas por parte de las profes, ellas deben saber cosas básicas. Por ejemplo, que las profes puedan generar procesos de indagación con respuestas acordes a lo que uno esperaría desde las ciencias. Digamos que si un niño le pregunta "¿Por qué el cielo es azul?", "¿Por qué el agua moja?", ella debería dar la explicación científica; preguntas muy de niños, pero que son preguntas de ciencia muy buenas que en ese momento con las profes de preescolar se pierden, porque no tienen ni idea de lo que deben contestar. (Docente 3)

Si bien durante la entrevista dos docentes indicaron esta necesidad, Ilama la atención que su comprensión estaba ligada a que no es posible enseñar ciencias si no se tiene la formación disciplinar, pues resulta esencial en la enseñanza de los conceptos. Es decir, identificaban como una barrera en la enseñanza de las docentes de preescolar su no formación disciplinar, 
sin reconocer sus capacidades de saber enseñar y el saber estar con los estudiantes (Lima y Maués, 2006).

Cuando los docentes cuestionan el paradigma con el que regularmente actúan y centran sus apuestas en las habilidades de pensamiento científico y tecnológico, y no en los contenidos disciplinares, se da paso a concepciones más sosegadas acerca de la apropiación de la ciencia y la tecnología en los grados de preescolar y primero. Al respecto, una docente de secundaria señala:

En un inicio, el contacto con los pequeños era mínimo, en estos años de docencia uno ha estado enfocado en los grandes. Cuando la Ruta nos hace pensar en los pequeños, el obstáculo es cómo vamos a hablar de ciencia y tecnología, ¿cómo acercarnos a ellos?, ¿cómo hacernos entender?, ¿cómo lo vamos a hacer? Nos dimos cuenta con el PIP de que los estudiantes de secundaria pueden ser el soporte y multiplicadores del aprendizaje, quienes al realizar las actividades se apropian más del saber en esa experiencia con los pequeñitos. La Ruta nos sirvió porque, en el momento de planear las actividades, ustedes nos dijeron que la hiciéramos para los grandes y para los pequeños, y ahí creo que fue donde nos abrimos y dijimos sí se puede no tan científico, no tan riguroso, más práctico, más desde lo cotidiano. (Docente 2)

Este conjunto de creencias muestra que, para las docentes, la enseñanza de las ciencias es una cuestión de contenidos, lo que se relaciona con la dimensión conceptual señalada por Delors (1996) y Lima y Maués (2006). Así, la preocupación de los docentes sobre los temas se relaciona con la cara de la ciencia como producto, propuesta por Furman (2008). Por tanto, se termina desconociendo las posibilidades que tienen las docentes cuando establecen relaciones de exploración del medio y de vivencia de experiencias, que permiten a niñas y niños vivir la ciencia como un proceso de formación de su pensamiento.

\section{Creencias sobre la disposición de las docentes hacia el conocimiento científico}

Otras concepciones en el proceso de la Ruta fueron identificadas por el equipo pedagógico de Maloka en una serie de disposiciones de las docentes de preescolar y primero que limitarían la apropiación social de la ciencia y la tecnología por parte de niñas y niños. En esa dirección, un miembro del equipo afirmó:

Las profes tienen un arraigo a lo que hacen, están en ese lugar y de ahí no se mueven. Al mismo tiempo, tienen problemas de tiempo, tienen otras responsabilidades, no tienen recursos ni materiales, por eso prefieren seguir haciendo lo que hacen. (Facilitadora 1)

Otra facilitadora señala que esa actitud pasiva no necesariamente tiene que ver con un no interés de las docentes, sino también con unos procesos de exclusión de ellas respecto a la ciencia y la tecnología en la escuela.

La profe tiene su espacio y no se ve articulada con otros, se queda en su nicho, en su aula, no hay articulación con lo que hay afuera, y eso les funciona, estar apartadas funciona y hacer lo que siempre hacen. No se ve articulada con otros espacios de la IED, como se hace en otros grados, que tienen otros espacios disponibles. Ellas no tienen la posibilidad de acceso a otros espacios, no se lleva a los niños al laboratorio porque no pueden tocar, solo mirar, son espacios restringidos para los niños pequeños, y no es porque ellas lo piensen, sino porque hay una 
cultura institucional en el que no es prioritario el acceso de los pequeños a los espacios de ciencia y tecnología de la IED, y a la final ellas se acostumbran al trabajo solo en su aula.

(Facilitadora 3)

En ese sentido, parece ser que la actitud pasiva de las docentes no solo tiene que ver con una decisión personal de no interés por el conocimiento científico, sino con la naturalización en las IED de que niñas y niños de educación inicial no requieren o no pueden o no están listos para los espacios de ciencia en la escuela. Así, por ejemplo, una persona del equipo de Maloka señala:

Una profe me dijo en un acompañamiento situado que la responsabilidad de la ciencia y la tecnología no era de ella, sino de los profesores de los grados superiores, entonces, que no entendía por qué en el marco de la Ruta debía trabajarlo. (Facilitador 4)

Este conjunto de creencias refleja nuevamente la comprensión de la ciencia como establecimiento firme y rígido en el que no hay cabida a la exploración, a la indagación de fenómenos o al lanzamiento de hipótesis, es decir, al vivir experiencias de ciencia. Lo que determina este accionar es precisamente esa concepción de ciencia y tecnología como una actividad rígida, la cual definen Fernández et al. (2002) como una concepción acumulativa del desarrollo científico, en donde solo es posible hacer ciencia en espacios destinados para ello. Además, surge como determinante en la apropiación de la ciencia y la tecnología una mirada de las ciencias como producto (Furman, 2008), que se debe adquirir en espacios dispuestos para estos conocimientos: Ios laboratorios. En consecuencia, las aulas de preescolar y primero se configuran como espacios no viables para vivir experiencias que promuevan el pensamiento científico y tecnológico.

\section{Consideraciones finales}

Después de este análisis, con base en las creencias identificadas en la Ruta de formación en ciencia y tecnología, es importante destacar tres puntos para futuros ejercicios de investigación, en aras de identificar posibles desafíos en la apropiación de la ciencia y tecnología por parte de la primera infancia.

En primer lugar, uno de los hallazgos más relevantes fue que la mayoría de las creencias identificadas en las docentes de preescolar y primaria reflejan visiones distorsionadas sobre la ciencia y tecnología, particularmente aquella mirada rígida de estas como un conocimiento exacto e infalible. Dicha concepción está en línea con la idea del conocimiento científico como producto, es decir, se cree que las niñas y niños se apropian de las ciencias y las tecnologías una vez aprenden determinados saberes o contenidos y no como un proceso en el que se desarrollan y potencian habilidades de pensamiento científico y tecnológico.

Esta circunstancia pone de manifiesto la necesidad de comprender con mayor profundidad cómo se configura esta creencia tanto en las maestras de educación inicial como en los demás actores de la comunidad educativa y cuáles serían los mejores caminos para superarla. En este punto es importante considerar cómo esos actores construyen estas concepciones, que relegan los espacios que no se consideran como propios del conocimiento científico y tecnológico. Así, las aulas de preescolar y primero no se perciben claramente como lugares para la ciencia, los laboratorios que sí son percibidos para tal fin no son considerados como 
aptos para niños y niñas de primeros años, ni estos últimos como capaces de hacer uso de ellos. En este punto es importante destacar que la ciencia se ve como un saber disciplinar delimitado en espacio y tiempo en la escuela, no como un saber que puede ser transversal a toda la experiencia educativa y, en general, a la manera en la que niñas y niños se relacionan con el mundo.

En segundo lugar, cabe resaltar la estrategia metodológica de la Ruta en sí misma, pues es una puerta a la transformación de las concepciones sobre el conocimiento científico en la primera infancia y su apropiación. En particular, la Ruta es un vehículo para transformar estas creencias encontradas, ya que las docentes de preescolar y primero confrontan sus concepciones sobre las posibilidades de aprendizaje de las ciencias y las tecnologías en niñas y niños. Esto ocurre a su vez con las docentes de ciencia y tecnología de los últimos grados de primaria y secundaria, quienes, al reconocer que tienen responsabilidad en los procesos de desarrollo y potenciamiento de habilidades científicas y tecnológicas de los infantes, identifican sus vacíos sobre el desarrollo integral de las infancias como la principal barrera para que los docentes promuevan relaciones de enseñanza/aprendizaje con preescolar y primer grado

Articulado con lo anterior, uno de los vacíos que más llama la atención tiene que ver con el desarrollo del saber pedagógico respecto a qué y cómo se enseña la ciencia y tecnología en preescolar y primero, ya que si bien existen políticas y lineamientos en educación y la enseñanza de las ciencias y la tecnología que podrían dar algunas respuestas, cada uno de estos frentes es una rueda suelta cuando se trata de infancia, es decir, hay una producción importante de orientaciones nacionales que les dicen a las maestras que la exploración del medio es importante en el desarrollo de las niñas y los niños; sin embargo, su énfasis es el desarrollo infantil, no el potenciamiento del pensamiento científico y tecnológico. Este aspecto se convierte en uno de los principales desafíos hallados en esta investigación, pues, el establecer puentes entre la comprensión del pensamiento científico y tecnológico y el saber pedagógico para potenciarlo en la vida de la escuela, es lo que permitirá avanzar en la apropiación de la ciencia y la tecnología en niñas y niños de 4 a 7 años.

Así ocurre también con las orientaciones sobre ciencia y tecnología; por ejemplo, en Colombia se encuentra que los derechos básicos de aprendizaje de las ciencias naturales (MEN, 2016 b) solo se ofrecen desde primero y están centrados en saberes específicos de las ciencias (una ciencia vista como producto), lo que deja de lado los procesos de desarrollo de pensamiento en los que se encuentran las personas a esta edad.

Esta disociación entre los procesos de apropiación social de las ciencias y la tecnología y la comprensión de la infancia se convierten en el desafío más relevante de formación docente hallada por la Ruta. En el día a día de la escuela, estos dos aspectos son una integralidad en las niñas y los niños, y si las docentes no cuentan con la formación específica para proponer experiencias que potencien el pensamiento científico y tecnológico, se continuará preservando la concepción de que hay que ser docente/científico para enseñar ciencias y tecnología, y cómo esa especie de docente solo se encuentra en la secundaria, las infancias seguirán siendo víctimas de estas brechas de enseñanza.

Finalmente, la Ruta demostró que los procesos de formación docente colaborativos, con la intención de construir conocimiento desde escenarios como los museos interactivos de ciencia y tecnología, son una posibilidad para cerrar las brechas en el desarrollo del conocimiento científico y su apropiación por parte de las infancias; de un lado, porque los proyectos de innovación permiten el trabajo interdisciplinar entre docentes y esto contribuye a la 
construcción de proyectos que beneficien a más niñas y niños. De otra parte, porque también apropiarse de los espacios de la ciudad, en este caso de Maloka, es una experiencia práctica que le enseña a la docente desde el hacer, específicamente desde el hacer de sus niñas y niños, quienes demuestran que están listos y que pueden aprender del mundo.

Desde la perspectiva de los museos interactivos, estos procesos de formación docente también le abren posibilidades y son capaces de poner en cuestión sus propios paradigmas y estructura. Vimos cómo el trabajo conjunto con docentes realizado en la Ruta deconstruyó los imaginarios que el equipo pedagógico del museo tenía sobre la primera infancia y, en este sentido, el equipo educativo del museo ganó mayores comprensiones para pensarse espacios en donde niñas y niños de primeros años de escolaridad exploren y aprendan sobre ciencia y tecnología.

\section{Referencias}

Arana, M., Sierra, L., Sequeda, S., Rodríguez, Y., Patarroyo, G., Ruíz, D., Agudelo, R. y Merchán, O. (2006). Informe final de investigación del proyecto DSISI-017-05 "Estrategias de educación científico y tecnológicas para el proceso de formación profesional de educadores infantiles en las universidades Pedagógica Nacional y Libre de Colombia”. CIUP.

Appleton, K. y Kindt, I. (2002). Beginning elementary teachers' development teachers of science. Journal of Science Teacher Education, 13(1), 43-61.

Barraza, A. (2005). Una conceptualización comprehensiva de la innovación educativa. Innovación Educativa, 5(28), 19-31.

Bachelard, G. (2000). La formación del espíritu científico. Siglo XXI Editores.

Beetlestone, J. G., Johnson, C. H., Quin, M. y White, H. (1998). The science center movement: context, practice, next challenges. Public Understand of Science, 1(7), 5-26. https://doi.org/10.1177/096366259800700101

Bonilla-Castro, E. y Rodríguez, P. (2005). Más allá del dilema de los métodos. La investigación en ciencias sociales. Grupo Editorial Norma - Uniandes.

Comisión Intersectorial de Atención Integral a Primera Infancia (CIPI). (2013). Fundamentos políticos, técnicos y de gestión. http://www.deceroasiempre.gov.co/Prensa/CDocumentacionDocs/Fundamientos-politicostecnicos-gestion-de-cero-a-siempre.pdf

Coombs, P. (1971). La crisis mundial de la educación. Ediciones Península.

De la Jara, I. (2015). Infancia, museo y memoria: voces insospechadas. Revista Cabás, 13, 70-91.

Delors, J. (1996). La educación encierra un tesoro. Informe a la Unesco de la Comisión Internacional sobre la Educación para el Siglo XXI. Ediciones Unesco.

Departamento Nacional de Planeación (DNP) y Colciencias. (2015). Manual metodológico general, para la identificación, preparación, programación y evaluación de proyectos.

Fernández, I., Gil, D., Carracosa, J. Cachapuz, A. y Praia, J. (2002). Visiones deformadas de la ciencia transmitidas por la enseñanza. Enseñanza de las Ciencias, 20(3), 477-488. 
Franco-Avellaneda, M. (2013a). Educación en museos: artefactos, conocimiento y sociedad. In C. Aguirre (Ed.), El museo y la escuela: conversaciones de complemento (pp. 37-52). Sello Explora - Editorial.

Franco-Avellaneda, M. (2013b). Ensamblar museus de ciências e tecnologias: compreensões educativas a partir de três estudos de caso [Tesis de doctorado]. UFSC.

Franco-Avellaneda, M. (2016). Trasferencia e intercambio: cuando el río suena... reflexiones para pensar el rumbo de la política de apropiación del conocimiento en Colombia. Trilogía. Ciencia, Tecnología y Sociedad, 8(15), 69-79.

Franco-Avellaneda, M. y Pérez-Bustos, T. (2009). ¿De qué ciencia hablan nuestros materiales de divulgación? Revista Colombiana de Educación, 1(56), 81-103.

Franco-Avellaneda, M. y Von-Linsingen, I. (2011). Popularizaciones de la ciencia y la tecnología en América Latina: mirando la política científica en clave educativa. Revista Mexicana de Investigación Educativa, 16(51), 1253-1272.

Freire, P. (2005). Pedagogía del oprimido. Siglo XXI Editores.

Furman, M. (2008). Ciencias naturales en la escuela primaria: colocando las piedras fundamentales del pensamiento científico. En IV Foro Latinoamericano de Educación, Aprender y Enseñar Ciencias. Desafíos, estrategias y oportunidades (pp. 1-21). Fundación Santillana.

Furman, M. (2016). Educar mentes curiosas: la formación del pensamiento científico y tecnológico en la infancia. Documento básico. En XI Foro Latinoamericano de Educación ( $p$. 88). Buenos Aires. Santillana.

Gallego, A. (2007). Imagen popular de la ciencia transmitida por los cómics. Revista Eureka sobre Enseñanza y Divulgación de las Ciencias, 4(1), 141-150.

Grossman, P. (1990). The making of a teacher. Teacher knowledge and teacher education. Teachers College, Columbia University.

Kaufmann, V. y Serulnicoff, A. (2000). Conocer el ambiente: una propuesta para las ciencias sociales y naturales en el nivel inicial. En A. Malajovich (comp.), Recorridos didáctico en la educación inicial (pp. 25-61). Paidós.

Kelly, J. (2000). Rethinking the elementary science methods course: A case for content, pedagogy, and informal science education. International Journal of Science Education, 22(7), 755-777.

Latour, B. (1987). Science in action. How to follow scientist and engineers through society. Harvard University Press.

Lima, M. y Maués, E. (2006). Uma Releitura do Papel da Professora das séries iniciais no desenvolvimento e aprendizagem de ciências das crianças. Revista Ensaio, 08(02), 184198.

Magnusson, S., Krajcik, J. y Borko, H. (1999). Nature, sources, and development of pedagogical content knowledge for science teaching. En J. Gess-Newsome y N. Lederman (eds.), Examining pedagogical content knowledge. The construct and its implications for science education (pp. 95-132). Kluwer Academic Publishers.

Maloka (2019a). Ruta de formación en ciencia y tecnología para instituciones educativas distritales. Documento orientador. 
Maloka (2019b). Ruta de formación en ciencia y tecnología para instituciones educativas distritales. Caracterización de docentes.

Maloka (2019c). Tercer informe de avance. Ruta de formación en ciencia y tecnología.

Massarani, L. y Moreira, I. de C. (2009). Ciência e público: reflexões sobre o Brasil. Redes. Revista de Estudios Sociales de La Ciencia, 15(30), 105-124.

Maués, E. (2003). Conhecimento pedagógico de conteúdo geral e o conhecimento de conteúdo de ciências das professoras das séries iniciais. UFMG.

Ministerio de Educación Nacional (MEN). (2014). La exploración del medio en la educación inicial. Documento N. ${ }^{\circ} 24$. Serie de orientaciones pedagógicas para la educación inicial en el marco de la atención integral. http://www.deceroasiempre.gov.co/Prensa/CDocumentacionDocs/Documento-N24exploracion-medio-educacioninicial.pdf?TSPD_101_R0=0883a32c4dab2000799ca963492037a40fca1aa2b82da53598ca 6ddd2153a86928331371becf329a082d51fe00143000d0a2bef70ffe0ee7b45414c7f418896 39ccc7560e55ea80f38797e249b805493bed5470429577ad8e962809174bf913c

Ministerio de Educación Nacional (MEN). (2016a). Derechos básicos de aprendizaje. V1. Transición.

https://aprende.colombiaaprende.edu.co/ckfinder/userfiles/files/DBA\%20Transición.pdf

Ministerio de Educación Nacional (MEN). (2016b). Derechos básicos de aprendizaje. Versión 1. Ciencias Naturales. https://aprende.colombiaaprende.edu.co/sites/default/files/naspublic/DBA_C.Naturales.p df

Ministerio de Educación Nacional (MEN). (2017). Bases curriculares para la educación inicial y el preescolar. Referentes técnicos para la educación inicial en el marco de la atención integral. Recuperado de: https://aprende.colombiaaprende.edu.co/sites/default/files/naspublic/DBA_C.Naturales.p df .

Parker, J. y Heywood, D. (2000). Exploring the relationship between subject knowledge and pedagogic content knowledge in primary teachers' learning about forces. International Journal of Science Education, 22(1), 89-111.

Pérez-Bustos, T. (2009). Tan lejos... Tan cerca. Articulaciones entre la popularizaciòn de la ciencia y la tecnología y los sistemas educativos en Colombia. Interciencia, 34(11), 814821.

Ruíz, D., Martínez, L. y Parga, D. (2009). Creencias de los profesores de preescolar y primaria sobre ciencia, tecnología y sociedad, en el contexto de una institución rural. Tecné, Episteme y Didaxis: TED, 25, 41-61.

Schiele, B. (2008). Science museums and science centers. En M. Bucchi y B. Trench (eds.), Handbook of public communication of science and technology (pp. 27-40). Routledge.

Screven, G. (1990). Uses of evaluation before, during and after exhibit design. International Laboratory for Visitor Studies, 1(2), 36-66.

Shulman, L. (1986). Paradigms and research programs in the study of teaching: A contemporary perspective. En M. Wittrock (ed.), Handbook of research on teaching (pp. 336). Macmillam. 
Thomas, H. (2009). Sistemas tecnológicos sociales y ciudadanía sociotécnica. Innovación, desarrollo, democracia. En T. M. Fernando y G. Gustavo (eds.), Culturas científicas y alternativas tecnológicas. I Encuentro Internacional (pp. 65-86). Ministerio de Ciencia, Tecnología e Innovación Productiva de Argentina.

Valbuena, E. (2007). El conocimiento didáctico del contenido biológico: estudios de las concepciones disciplinares y didácticas de futuros docentes de la Universidad Pedagógica Nacional (Colombia) [Tesis doctoral]. Universidad Complutense de Madrid.

Yager, R. y Penick, J. (1983). Analysis of the current problems with school science in the United States of America. European Journal of Science Education, 5(4), 463-469. 